\title{
Flow around a hemisphere-cylinder at high angle of attack and low Reynolds number. Part II: POD and DMD applied to reduced domains
}

\author{
Soledad Le Clainche ${ }^{\mathrm{a}} \quad$ Daniel Rodríguez ${ }^{\mathrm{a}}$, Vassilis Theofilis ${ }^{\mathrm{a}}$, Julio Soria ${ }^{\mathrm{b}, \mathrm{c}}$ \\ ${ }^{\text {a }}$ School of Aeronautics, Universidad Politécnica de Madrid, Spain \\ ${ }^{\mathrm{b}}$ Laboratory for Turbulence Research in Aerospace and Combustion, Department of Mechanical and Aerospace Engineering, Monash University, Australia \\ ${ }^{c}$ Department of Aeronautical Engineering, King Abdulaziz University, Saudi Arabia
}

\begin{abstract}
A B S T R A C T
Three-dimensional direct numerical simulations (DNS) have been performed on a finite-size hemispherecylinder model at angle of attack $A o A=20^{\circ}$ and Reynolds numbers $R e=350$ and 1000 . Under these conditions, massive separation exists on the nose and lee-side of the cylinder, and at both Reynolds numbers the flow is found to be unsteady. Proper orthogonal decomposition (POD) and dynamic mode decomposition (DMD) are employed in order to study the primary instability that triggers unsteadiness at $\mathrm{Re}=350$. The dominant coherent flow structures identified at the lower Reynolds number are also found to exist at $\operatorname{Re}=1000$; the question is then posed whether the flow oscillations and structures found at the two Reynolds numbers are related. POD and DMD computations are performed using different subdomains of the DNS computational domain. Besides reducing the computational cost of the analyses, this also permits to isolate spatially localized oscillatory structures from other, more energetic structures present in the flow. It is found that POD and DMD are in general sensitive to domain truncation and noneducated choices of the subdomain may lead to inconsistent results. Analyses at $\operatorname{Re}=350$ show that the primary instability is related to the counter-rotating vortex pair conforming the three-dimensional afterbody wake, and characterized by the frequency $S t \approx 0.11$, in line with results in the literature. At $\operatorname{Re}=1000$, vortex-shedding is present in the wake with an associated broadband spectrum centered around the same frequency. The horn/leeward vortices at the cylinder lee-side, upstream of the cylinder base, also present finite amplitude oscillations at the higher Reynolds number. The spatial structure of these oscillations, described by the POD modes, is easily differentiated from that of the wake oscillations. Additionally, the frequency spectra associated with the lee-side vortices presents well-defined peaks, corresponding to $\mathrm{St} \approx 0.11$ and its few harmonics, as opposed to the broadband spectrum found at the wake.
\end{abstract}

\section{Introduction}

The hemisphere-cylinder is a model geometry for the study of the aerodynamic properties of air-to-air missiles, vehicles of high maneuverability that can be subjected to very high angles of attack (AoA). Massive three-dimensional separation leading to complex flow patterns is one of the main difficulties found in the design and improvement of the existing methods for the prediction and control of aerodynamic performances. Potential flow methods used traditionally in aircraft industry invariably fail when flow separation occurs, and the ad hoc modifications employed to account for two-dimensional separation cannot be extended to the complex flow topologies associated with massive separation from three-dimensional bodies. Massive flow separation is also related to the appearance of important oscillations on the force, drag and momentum coefficients, affecting vehicle stability and maneuverability.

Several studies have addressed the complex flow topology patterns that form over the hemisphere-cylinder geometry under flow separation conditions $[3,7,9,15,17,38]$. Three different kinds of flow separation were found: (a) flow separation occurring around the hemispherical nose, due to the longitudinal pressure gradient induced by the geometry and the angle of attack, (b) separation of the boundary layer around the cylinder body due to the transversal pressure gradient, giving rise to the leeward vortices, and (c) bluff body separation in the cylinder flat base. In addition, the flow in the nose separation region can be organized into different topologies, referred to as closed laminar separation bubble, horseshoe 
vortex or horn-vortices. The topology of the separated flow around the hemisphere cylinder geometry was discussed in a preceding paper [20], and in Le Clainche [19].

The physical mechanisms responsible for the oscillatory forces associated with massive separation, and the particular flow structures associated with them are another open question regarding the hemisphere-cylinder geometry. Based on the research done on the oscillations on plane mixing layers $[8,10]$ and past circular cylinders [24], it can be expected that the linear hydrodynamic instability mechanism responsible for the onset of unsteadiness at low Reynolds number can also explain the dominant frequencies observed at higher Reynolds numbers. Bohorquez et al. [5] and Sanmiguel-Rojas et al. [33] performed direct numerical simulations and linear stability analysis of the hemisphere-cylinder geometry at $\mathrm{AoA}=0^{\circ}$. A global linear instability eigenmode was found to be responsible for the oscillations of the counter-rotating vortex pair forming the three-dimensional wake. This instability, initially leading to peristaltic motions in the wake vortices and to threedimensional vortex shedding at higher Reynolds numbers, had an associated non-dimensional frequency St $\approx 0.12$. The same instability mechanism, including its dimensionless frequency, was also found to dominate the wake past three-dimensional bluff bodies [39]. Gross et al. [13] performed direct numerical simulations and experiments at higher Reynolds numbers ( $\operatorname{Re}=2000$ and 5000) and moderate-to-high angles of attack, focusing the attention on the nose separated flow and its unsteadiness. They pointed out the existence of structures and frequencies in the near-nose region and associated them to an inviscid instability of the nose-separation shear layer. They also suggested that the variations observed in the flow structures with changes in angle of attack and Reynolds numbers can be associated with different linear instability mechanisms: at $\mathrm{AoA}=10^{\circ}$ and $\mathrm{Re}=5000$ they observe structures related to the instability of the separated shear layer at the nose, originated by the longitudinal pressure gradient; increasing the angle of attack to $30^{\circ}$ resulted in a change of the near-nose flow topology, giving rise to a pair of horn vortices and the reduction in size of the separation bubble. In this case, they noted the appearance of azimuthally aligned structures and related them to the instability of the separated shear-layer associated with the transversal pressure gradient.

The work presented in Le Clainche et al. [20] considered a hemisphere-cylinder geometry at $\mathrm{AoA}=20^{\circ}$ and $\operatorname{Re}=1000$, and combined experimental measurements using time-resolved planar PIV on a water tunnel and three-dimensional direct numerical simulations (DNS). Two techniques for the extraction and analysis of coherent flow structures existing in empirical (either from experimental or from numerical simulations) data sets, namely proper

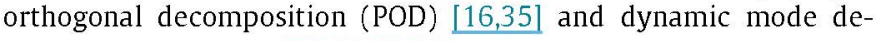
composition (DMD) $[22,23,31,34]$, were employed to study the dominant fluctuations on the lee-side of the cylinder. A welldefined peak frequency of $\mathrm{St} \approx 0.12$ was identified and the corresponding spatial structures obtained by means of POD and DMD were nearly identical. Interestingly, the domain employed in the POD/DMD analyses was a subdomain extracted from the total DNS computational domain, such that the wake and its influence on the global flow characteristics were removed. While the existing literature $[5,39]$ agrees that the St $\approx 0.12$ is related to wake oscillations, the analysis of Le Clainche et al. [20] recovers the same frequency on oscillations associated with the lee-ward vortices over the cylinder, upstream of the base and the wake. The question remains whether this result is imprinted on the global flow instability characteristics.

The present work revisits the application of POD and DMD techniques for the study of the dominant flow structures existing in the hemisphere-cylinder geometry, with two main objectives. The first one is concerned with the analysis techniques themselves, and their capacity to extract the dominant flow structures and frequencies from DNS data when a reduced subdomain is used, instead of the complete computational domain. The large computational cost of applying POD/DMD to data from time-resolved threedimensional simulations often makes it necessary to downsample the data and extract subdomains, in order to make the problem tractable with the hardware available. In some cases, it can also be interesting to isolate a particular spatial region in which a distinct flow behavior exists. Provided that the computational domain and resolution in the DNS computations is large enough to capture adequately the physics, a posteriori downsampling and domain truncation of the data does not alter the inherent physics, but can modify the manner in which POD and DMD captures them.

A deeper understanding of this aspect will shed light on the results of Le Clainche et al. [20], and help to address the second objective of the present work: determining the physical mechanism responsible for the onset of unsteadiness (the primary instability) and the dominant frequencies present in this flow configuration at higher Reynolds numbers, and elucidating if they are related to each other. With this aim, two different Reynolds numbers, Re = 350 and $\operatorname{Re}=1000$, are analyzed using POD and DMD. $\operatorname{Re}=350$ is slightly above the critical Reynolds number for the onset oscillations, being representative of the weakly-nonlinear saturated flow close to a Hopf bifurcation. A strong similarity between the leading linear instability eigenmode and the recovered POD/DMD modes is expectable at these conditions, in view of other results in the literature that will be discussed in the next paragraphs. At $\mathrm{Re}=1000$, a strongly nonlinear vortex shedding exists in the afterbody wake, but organized oscillations also exist in the lee-side of the cylinder [20].

The relation between linear instability and the structures recovered from POD/DMD analyses of nonlinearly saturated flows is another open question in the literature. The works of Rempfer and Fasel $[27,28]$ were the first to relate POD eigenfunctions with amplitude functions computed by linear stability theory (LST), corresponding to Tollmien-Schlichting waves in transitional data on a flat-plate boundary layer. Rowley et al. [29,30] applied POD and LST to compressible open cavity flows. They found certain analogies in the spatial structures of the leading POD modes with those of the locally-parallel LST in the unstable shear-layer region. Sengupta et al. [37] also found qualitative analogies between LST and POD results in the analysis of the laminar flow past a circular cylinder. Merzari et al. [21] and Oberleithner et al. [25] applied POD analysis to the turbulent flows in an annular duct, and found that the leading POD modes also exhibit strong spatial similarities with the amplitude functions of the respective leading LST eigenmodes. However, these successes are attributable to the predominance of a single dominant Fourier frequency in the flow dynamics. In a more complex flow, Tammisola et al. [36] compared the results obtained using global linear stability analysis on the linear regime with the frequency obtained in the nonlinear saturated regime on a viscous plane wake, finding substantial differences.

On the other hand, the spectral theory of the Koopman operator $[22,23,31]$ on which DMD is based, shows that when DMD is applied to a data set obtained from the solution of the linearized Navier-Stokes equations, DMD modes reduce to linear instability eigenmodes. Also, when DMD is applied to time-periodic flows, i.e. most of the nonlinear saturated flows, the results of DMD are equivalent to those from a temporal discrete Fourier transform of the data. Chen et al. [6] and Bagheri [1] applied DMD to study the flow around a circular cylinder in nonlinear saturated flows. They identified five dominant DMD modes, one steady and two pairs of complex conjugates corresponding to the fundamental and first harmonic frequencies, a description analogous to the POD-based reduced order model by Noack et al. [24]. On nonlinear flows, close to a Hopf bifurcation where the flow contains a dominant 
structure and frequency, the spatial structures recovered by LST, DMD and POD are very similar, but the frequencies recovered can differ, as observed by Rowley et al. [31] in the study of a jet in a crossflow. Finally, Gómez et al. [12] compared LST results obtained using global instability analysis based on a time-stepping technique with POD and DMD modes, finding a good agreement between all these techniques in recovering the leading LST eigenmode in a lid-driven cavity flow.

The rest of the paper is organized as follows: Section 2 explains the basic theory and the POD and DMD algorithms employed for this research. Section 3 briefly explains the DNS code and the flow conditions and shows the computational domain in which the analyses were performed. Finally, Section 4 and Section 5 show the main results and conclusions, respectively.

\section{Theory}

\subsection{Proper orthogonal decomposition}

Proper orthogonal decomposition (POD) $[16,35]$ was first introduced into fluid mechanics for the extraction of the large-scale coherent structures existing in turbulent flows, and later applied to the construction of reduced order models [24,29] of complex flow behavior. POD is a statistical technique that delivers the basis of time-independent orthogonal functions $\psi^{(k)}(\mathbf{x})$ (known as POD modes) that optimally represents a given flow field or data set $\mathbf{u}(\mathbf{x}, t)$. The optimality condition is that the least square error of the truncated flow field expansion by the functions $\psi^{(k)}$,

$\mathbf{u}^{*}(\mathbf{x}, t)=\sum_{k=1}^{K} a_{k}(t) \psi^{(k)}(\mathbf{x})$

and the empirical (or exact) flow field $\mathbf{u}(\mathbf{x}, t)$ is minimized. The time-dependent scalars $a_{k}(t)$ are obtained from the projection of the POD modes on the empirical data set and are known as the POD coefficients. As measure, the standard inner product for vector functions is employed:

$\langle\mathbf{f}(\mathbf{x}), \mathbf{g}(\mathbf{x})\rangle=\int_{D} \mathbf{f}(\mathbf{x}) \cdot \mathbf{g}(\mathbf{x}) d \mathbf{x}$,

where $D$ is the three-dimensional integration domain. In the present case this inner product corresponds to the kinetic energy norm, as the vector function $\mathbf{u}(\mathbf{x}, t)$ is the velocity vector.

It can be shown [16] that the optimization problem for $\psi^{k}$ leads to the eigenvalue problem

$\mathbf{R} \psi^{(k)}=\lambda^{k} \psi^{(k)}$,

where $\lambda^{k}$ are the POD eigenvalues and $\mathbf{R}$ is a correlation matrix defined using the empirical data. In the classic approach of Holmes et al. [16], $\mathbf{R}$ is defined as the ensemble average of the correlation matrix defined between velocity components at different spatial locations, at each realization or snapshot. This leads to a correlation matrix with leading dimension equal to the number of discretization points times the number of velocity components, which becomes prohibitively large when a three-dimensional flow field is considered. Here, the snapshots method introduced by Sirovich [35] is employed instead, that results in a considerably smaller eigenvalue problem. The element $\tilde{R}_{i j}$ of the new correlation matrix is computed as the correlation of two different realizations, using the inner product (2):

$\tilde{R}_{i j}=\left\langle\mathbf{u}^{(j)}, \mathbf{u}^{(i)}\right\rangle$,

where $\mathbf{u}^{(i)}=\mathbf{u}\left(\mathbf{x}, t_{i}\right)$. Using the snapshots method, the correlation matrix $\mathbf{R}$ has leading dimension equal to the number of snapshots employed, typically orders of magnitude smaller than the number of discretization points where numerical simulation data is used. The eigenvalue problem now becomes

$\tilde{\mathbf{R}} \beta^{k}=\lambda^{k} \beta^{k}$,

and the POD modes are retrieved from

$\psi^{(k)}=\sum_{l} \beta_{l}^{k} \mathbf{u}^{(l)}$

POD modes are orthogonal under the inner product used, so the empirical data can be expanded as a linear combination of the POD modes (1), with

$a_{k}\left(t_{i}\right)=\left\langle\psi^{(k)}, \mathbf{u}^{(i)}\right\rangle /\left\langle\psi^{(k)}, \psi^{(k)}\right\rangle$

By construction, the ensemble average of $a_{k}(t)$ verifies $\overline{a_{k}}=\sqrt{\lambda^{k}}$. The POD eigenvalues thus constitute a measure of the statistical significance of each POD mode on the empirical data set, under the inner product considered. In the present case, $\lambda^{k}$ is a measure of the fluctuating kinetic energy associated with POD mode $\psi^{(k)}$. Due to the properties of the correlation matrix, $\lambda^{k}$ constitutes a set of non-negative eigenvalues that can be ordered according to their value, so that $\lambda^{1}>\lambda^{2}>\cdots>\lambda^{N}>0$. In this manner, the first POD mode is the one associated with the highest kinetic energy. A common practice in studies employing POD for reduced order modeling, is to show the relative significance of the POD modes in a truncated series expansion

$E(\%)=\frac{\lambda^{i}}{\sum_{i=1}^{N} \lambda^{i}} \cdot 100$.

In those cases in which a few number of POD modes account for a large percentage of the total kinetic energy $E(\%)$, these POD modes constitute a good approximation to the total flow field.

The POD analysis presented so far determines the spatial structures (POD modes) dominant in the flow field, as well as their ensemble-averaged kinetic energy. More information can be extracted by monitoring the temporal evolution of the POD coefficients, $a_{k}(t)$ for the dominant modes. This analysis is done here in the frequency domain, in order to allow comparisons with the results from DMD. The Power Spectral Density distribution (PSD) (or power spectra) of each $a_{k}(t)$ is computed using one-sided Fast Fourier Transform and a rectangular window with half-overlapping segments, in order to reduce the variance of the computed spectra.

\subsection{Dynamic mode decomposition}

The dynamic mode decomposition (DMD) technique has recently been introduced [34] for the approximate computation of Koopman modes $[22,23,31]$, providing a tool for the analysis and reduction of linear and nonlinear dynamical systems, and in the present case, unsteady flow fields. The DMD algorithm is briefly described next.

Assuming the existence of a linear mapping $A$, constant over the snapshots sequence that connects the flow field $\mathbf{u}^{(i)}$ with the subsequent flow field $\mathbf{u}^{(i+1)}$, the following Krylov series can be defined:

$U_{1}^{N}=\left\{\mathbf{u}^{(1)}, A \mathbf{u}^{(1)}, A^{2} \mathbf{u}^{(1)}, \ldots, A^{N-1} \mathbf{u}^{(1)}\right\}$.

Given a data set consisting of $(N+1)$ snapshots resolved in time, two different snapshots matrices can be constructed: $U_{1}^{N}$ (from the first to the $N$ th snapshot) and $U_{2}^{N+1}$ (from the second to the $(N+1)$ th snapshot). For a long data snapshots sequence, any further snapshots sequence can be expressed by linear combination of the previous ones: 
$U_{2}^{N+1}=A U_{1}^{N} \simeq U_{1}^{N} S+r e_{N-1}^{T}$

where $S$ is a companion matrix, $r$ is the residual and $e_{N-1}$ the $(N-1)$ th unit vector. The residual vector is minimized via the solution of the eigenvalue problem of matrix $S$, the eigenvalues of which are good approximations to those of the operator $A$. DMD modes are then determined by the projection of the snapshots matrix $U_{1}^{N}$ over the eigenvectors of the companion matrix $S$. Note that the linear operator $A$ is never required and the DMD eigenvalues and modes $\left(\omega^{k}\right.$ and $\Phi^{(k)}(\mathbf{x})$, respectively) are obtained directly from the empirical snapshots. The real part of the eigenvalue, $\omega^{k}=2 \pi f$, is a circular frequency, while the imaginary part is an exponential growth rate.

As opposed to POD modes, in which spatial orthogonality of the modes is imposed, DMD imposes temporal orthogonality: the sequence of snapshots in the empirical data need be acquired with a constant sampling interval $\Delta t$. Then, considering a linear or time-periodic flow, it is possible to define a time-shift between two snapshots based on a particular frequency $f: K=$ $\{1, \exp (2 \pi$ if $\Delta t), \exp (4 \pi$ if $\Delta t), \cdots\}$. From this follows that when DMD is applied to a flow field resulting from the solution of the linearized Navier-Stokes equations, DMD modes reduce to linear global modes [12] and when DMD is applied to time-periodic flows, DMD modes reduce to Fourier modes [6,31], in agreement with the spectral properties of the Koopman operator $[22,23,31]$

The flow fields considered in this work correspond in all cases to nonlinear saturated flows for which the temporal behavior can be associated to a periodic oscillatory flow, as will be shown in Section 4. In cases like this, the recovered DMD eigenvalues have a real frequency and constant amplitude. In order to rank the DMD modes and compare with results from POD, the integrated kinetic energy norm is also employed here:

$\left\|\Phi^{(k)}\right\|^{2}=\left\langle\Phi^{(k)}, \Phi^{(k)}\right\rangle$.

\section{Numerical computations}

\subsection{Direct numerical simulations}

Three-dimensional direct numerical simulations (DNS) are performed to study the flow around a hemisphere-cylinder of aspect ratio $L / D=8$ ( $L$ is the length of the cylinder forming the body and $D$ is the diameter) at angle of attack AoA $=20^{\circ}$ and three Reynolds numbers: 300, 350 and 1000. The diameter $D$ and the free-stream velocity $U_{\infty}$ are used to non-dimensionalize lengths and velocities, leading to the Reynolds number $\operatorname{Re}=U_{\infty} D / \nu$, where $v$ the kinematic viscosity. Consequently, the time is nondimensionalized with the unit time $D / U_{\infty}$. The onset of unsteadiness was found to take place for a Reynolds number between 300 and 350 [19].

The numerical code used for the DNS is OpenFOAM (http:// www.openfoam.com), an open source Computational Fluid Dynamics software that discretizes the incompressible Navier-Stokes equations using a finite volume method. A second order central differencing scheme (for the convective and diffusive term) is used for the spatial discretization and a second order Crank-Nicholson scheme discretizes the temporal term. High-quality flow stability results over axisymmetric bodies have been obtained recently using this code by Bohorquez et al. [4,5] and Sanmiguel-Rojas et al. [32,33].

The computational domain employed is a coaxial cylindrical surface of diameter $20 D$ and $50 D$ long. Le Clainche et al. [19,20] showed that this length was sufficient to accurately capture the physics of this problem at similar flow conditions. The mesh employed is composed of a regular tetrahedral grid with $\sim 2.8$ million cells and was generated using the OpenFOAM utility blockMesh.
Table 1

POD and DMD analyses computational subdomains.

\begin{tabular}{ll}
\hline Subdomain & Number of cells \\
\hline$D_{1}$ & 543400 \\
$D_{2}$ & 246400 \\
$D_{3}$ & 123200 \\
\hline
\end{tabular}

To avoid numerical singularities, the mesh within the sphere region was created following the numerical algorithm that defines a cubic sphere [26].

No-slip boundary conditions are imposed on the body surface:

$\mathbf{u}(\mathbf{x}, t)=\mathbf{0}, \quad \partial_{n} p(\mathbf{x}, t)=0$,

and free stream boundary conditions are used on the far-field, with $U_{\infty}=1$. This amounts to imposing

$\mathbf{u}(\mathbf{x}, t)=\left(U_{\infty} \cos (A \circ A), U_{\infty} \sin (A \circ A), 0\right)$,

$\partial_{n} p(\mathbf{x}, t)=0$

at the inlet and lateral sides of the domain, and

$\partial_{n} \mathbf{u}(\mathbf{x}, t)=\mathbf{0}, \quad p(\mathbf{x}, t)=0$

at the outlet.

The validation of the code was presented elsewhere [19,20], in which the numerical simulations where compared with literature results [32] and with time-resolved particle image velocimetry (PIV) experiments. The DNS simulations featuring this mesh topology were shown to be close to grid-independence, where a numerical error of $\sim 3 \%$ for the integral quantities in saturated flow was estimated.

\subsection{Data set domain truncation for POD and DMD analyses}

Proper orthogonal decomposition (POD) and dynamic mode decomposition (DMD) are two techniques commonly employed in the analysis of complex flow behavior and identification and extraction of the dominant flow structures from empirical flow-field data sets, obtained either experimentally or by numerical simulation. One of the alleged advantages of these techniques is that they can be applied to reduced portions of the spatial domain in which the empirical data is given, and still they provide accurate results while reducing the computational cost. This is of particular interest when considering data from three-dimensional DNS, as both the resolution and the domain size can be drastically reduced. POD and DMD are expected to deliver consistent results in this case because the true physics, involving the need for large resolution and domain extent, are already introduced in the computation of the data set. One of the objectives of the present work is to study the effect of the domain truncation on the results of POD and DMD approaches when applied to the flow around the hemisphere-cylinder geometry.

Three subdomains of the DNS data, $D_{1}, D_{2}$ and $D_{3}$, are extracted for the analysis. In the subdomain $D_{1}$, the radial extent of the domain is reduced to the half $(10 D)$ and the spatial locations with negative $Y$ component have been removed. This subdomain contains the separated flow on the hemisphere-cylinder lee side and most of the wake. Subdomain $D_{2}$ results from $D_{1}$ when the volume downstream of the body is also removed. In this case, the objective is to focus the analysis on the flow separation region and most of the wake is eliminated. Finally, the third subdomain $D_{3}$ is similar from $D_{2}$, but the radial extent is reduced to $5 D$ and the azimuthal domain is reduced to comprise only a 90 degree wedge. This subdomain contains the nose separated flow and the leewardvortices separation. Fig. 1 shows the three different subdomains. 

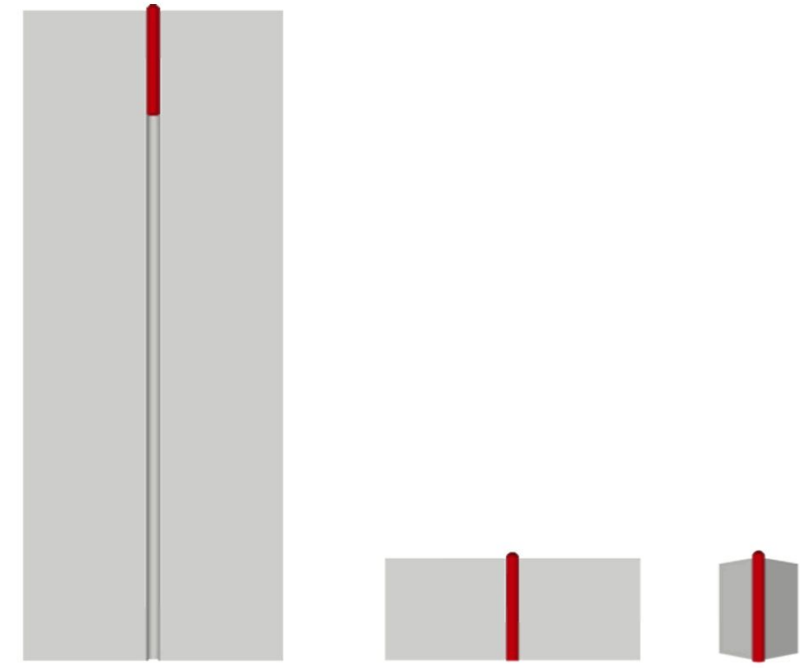

(a)

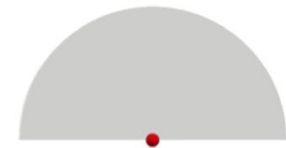

(b)

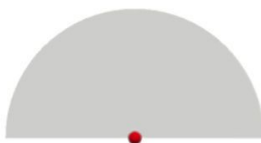

(c)

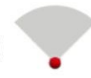

Fig. 1. Computational subdomains for POD and DMD analyses. Hemisphere-cylinder is shown in red and computational domain in grey: (a) Subdomain $D_{1}$. (b) Subdomain $D_{2}$. (c) Subdomain $D_{3}$. (For interpretation of the references to color in this figure legend, the reader is referred to the web version of this article.)

\section{Table 2}

Number of snapshots $N$ and corresponding frequency bin $\triangle S t$ employed in the POD and DMD analyses. $\Delta t=0.005$ in all cases.

\begin{tabular}{llll}
\hline Re & Analysis & $N$ & $\Delta$ St \\
\hline 350 & POD & 150 & 0.027 \\
& DMD & 80 & 0.021 \\
\multirow{2}{*}{1000} & POD & 150 & 0.027 \\
& DMD & 120 & 0.013 \\
\hline
\end{tabular}

The DNS data set is downsampled to a rougher mesh containing $\sim 1.5 \times 10^{6}$ cells prior to extracting the data on the three subdomains, in order to reduce the computational cost [2]. Table 1 compares the final grid sizes for each computational subdomain.

\section{Results}

Three-dimensional DNS were presented in Le Clainche [19] for the hemisphere-cylinder geometry with aspect ratio $L / D=8$ at $\mathrm{AoA}=20^{\circ}$ and different Reynolds numbers. The flow was found to be steady for $\operatorname{Re} \leq 300$, and simulations were also conducted at $\operatorname{Re}=350$ and 1000 , conditions at which the flow is unsteady. In both cases, flow separation existed in the nose region, generated by the longitudinal pressure gradient, as well as lee-ward vortices due to the transversal pressure gradient [19]. Different topological descriptions in the nose regions were observed for $\operatorname{Re}=350$ and 1000 , as well as a more complex flow field associated with the higher Reynolds number.

The simulations corresponding to $\mathrm{Re}=350$ and 1000 were marched until a nonlinear saturated regime was attained, and then continued further in time in order to compute the data set to be used in the POD/DMD analyses. Then, the data was downsampled and extracted to the three subdomains $D_{1}, D_{2}$ and $D_{3}$, as explained in the previous section, in order to make the POD and DMD approaches tractable.

Convergence tests are done by comparing the energy content of the four most relevant POD and DMD modes as a function of the number of snapshots. It is found that $N=120$ snapshots

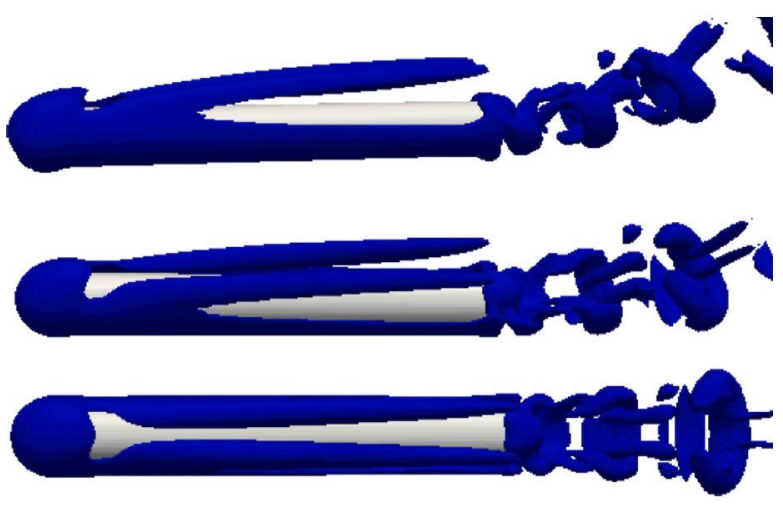

Fig. 2. Iso-surfaces of instantaneous $Q=300$ at $\operatorname{Re}=350$ and $A o A=20^{\circ}$.

and a sampling interval $\Delta t=0.005 \mathrm{~s}$ permits a relatively consistent recovery of the dominant POD and DMD eigenvalues and modes. As explained in Section 2, a frequency analysis of the POD coefficients $a_{k}(t)$ is also performed to enable comparisons with the DMD results in the frequency domain. The sampling theory for discrete Fourier analysis dictates a relation between the frequency bin $\Delta f, \Delta t$ and the number of samples (i.e. snapshots) employed $N, \Delta f=1 /(\Delta t \cdot N)$. Consequently, the spectra corresponding to the POD coefficients is discretized in frequency bars $\Delta f$, the width of which is a compromise between the number of snapshots and the sampling interval. It is interesting to note that when applying the DMD algorithm to the present data set, all the DMD eigenvalues recovered correspond to real frequencies which are equispaced, and their spacing matches exactly the frequency bin for the PSD computation associated with the $\Delta t$ and $N$ employed. In what follows, frequencies are expressed by the Strouhal number $\mathrm{St}=f \sin (\mathrm{AoA}) U_{\infty} / D$. Table 2 shows the number of snapshots used in the different analyses and their corresponding PSD frequency bin $\Delta S t$. In all the cases, $\Delta t=0.005$ time units.

4.1. Case 1: $\operatorname{Re}=350, A \circ A=20^{\circ}$

For the hemisphere-cylinder geometry at $\mathrm{AoA}=20^{\circ}$, unsteadiness appears first related to the wake at a Reynolds number between $\operatorname{Re}=300$ and 350 . At $\operatorname{Re}=350$, a weakly-nonlinear saturated regime exists, with small but finite amplitude oscillations in the afterbody and wake region. However, the nose separation bubble is nearly steady under these conditions. Consequently, the analysis in subdomain $D_{3}$, centered in the nose and lee-side of the cylinder, was not performed for this case.

Fig. 2 shows instantaneous coherent structures detected using $Q$-criterion method [18]. Iso-surfaces corresponding to $Q=300$ are shown, permitting the identification of three distinct flow regions: the separation bubble on the hemispheric nose region, the lee-ward vortices formed by the separation of the boundary layer on the cylinder sides, and the wake region in which a regular pattern of shed vortices is observed.

Fig. 3(a, left) shows the relative kinetic energy (in percentage) associated with the 10 most relevant POD modes computed in domain $D_{1}$. As usual in POD analyses of oscillatory flows, the POD modes appear in couples, representing traveling structures or waves: the first and second POD modes have similar energy and together account for more than $50 \%$ of the total fluctuating kinetic energy; the third and fourth also have similar energy and account for over $24 \%$ of the total. Modes five and six comprise around $15 \%$ of the fluctuating kinetic energy. In total, the first six POD modes contain about $90 \%$ of the energy, implying that the flow is dominated by strongly coherent structures. The power spectra corresponding to the POD coefficients of the first six modes is shown in Fig. 3(a, right). It should be remarked that, while the num- 

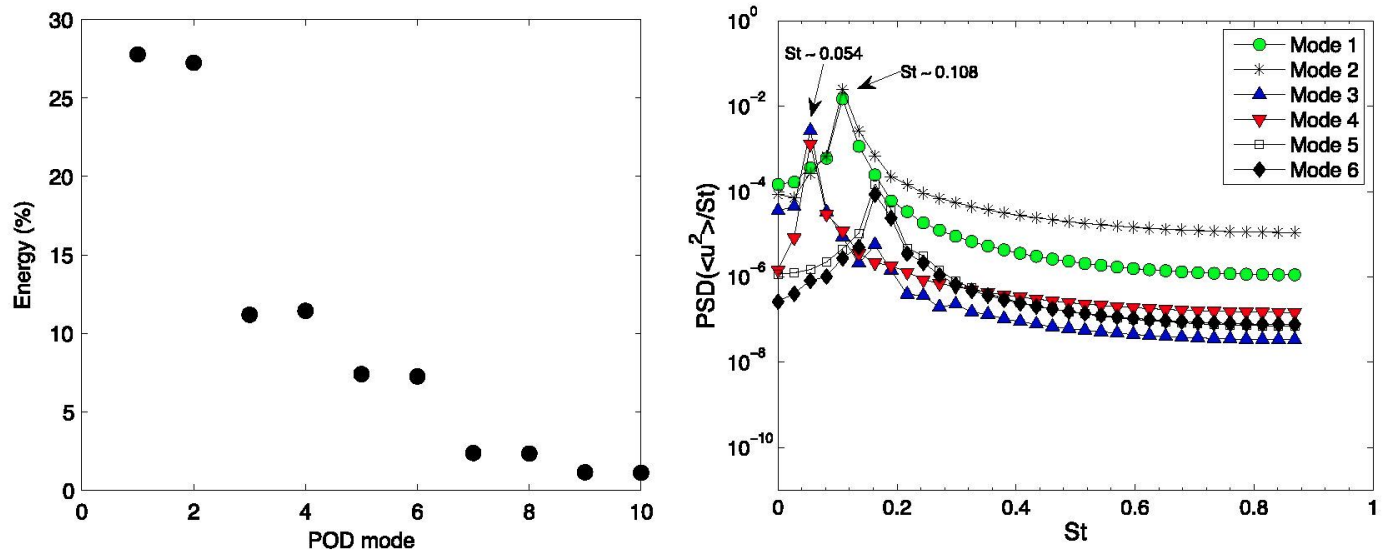

(a) POD in subdomain $D_{1}$
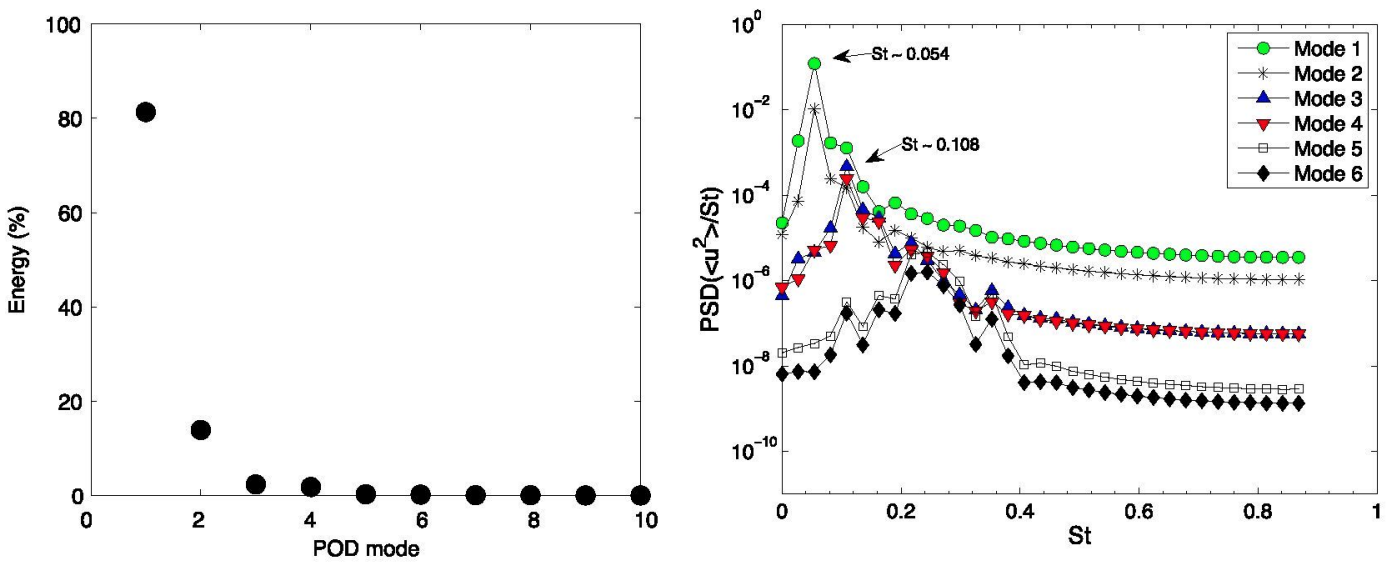

(b) POD in subdomain $D_{2}$
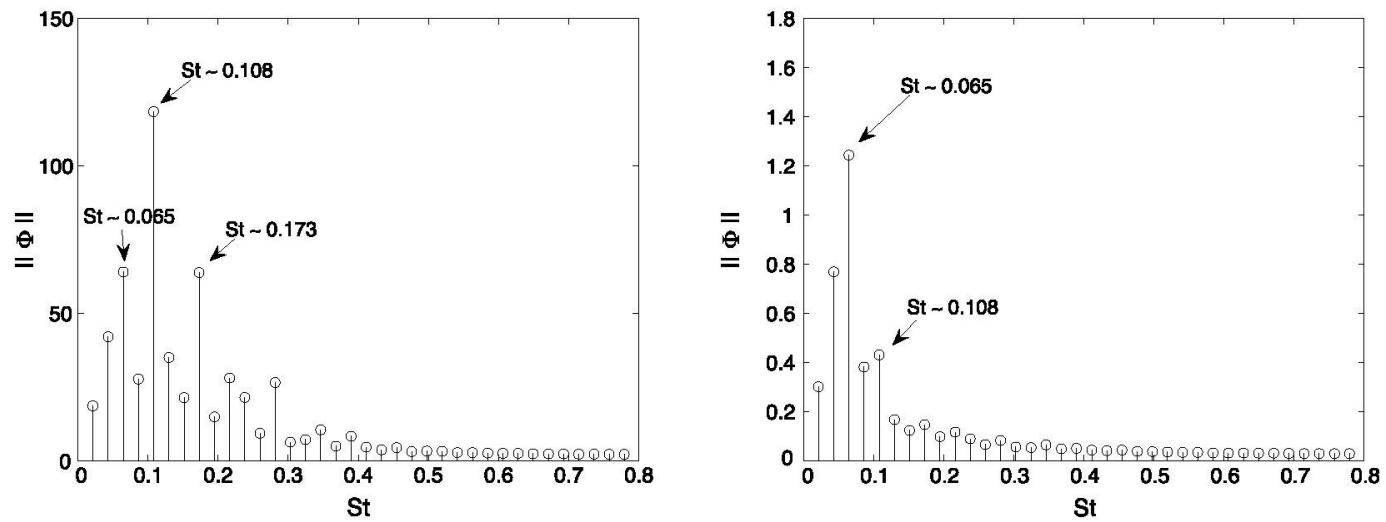

(c) DMD in subdomains $D_{1}$ and $D_{2}$

Fig. 3. POD analysis in subdomains $D_{1}$ (a) and $D_{2}$ (b) at $R e=350$ and AoA $=20^{\circ}$. Kinetic energy distribution (left) and PSD of POD coefficients (right). (c) DMD frequency spectra in domains $D_{1}$ and $D_{2}$

ber of snapshots was shown to be enough to achieve convergence of the POD modes, it is small to ensure convergence of the PSD. First and second modes are dominated by the single frequency $\mathrm{St}=0.108 \simeq 0.11$. The same frequency (due to the frequency bin $\Delta S t=0.027$, the actual frequency is $S t=0.108 \pm 0.014$ ) has been found for the hemisphere-cylinder at low Reynolds number and zero angle of attack [5] and flow around bluff bodies [39]. In all cases, this frequency was clearly associated with oscillations of the three-dimensional wake, first as a peristaltic instability of the counter-rotating vortices [14], and then as vortex shedding when the Reynolds number is increased. The third and fourth modes are associated with the sub-harmonic frequency, St $=0.054$. Finally, a frequency about $\mathrm{St}=0.23$ is obtained for modes five and six, with spectral amplitude two orders of magnitude smaller than that of the fundamental.

Fig. 3(b) shows the relative energy content of the POD modes and their frequency spectra, obtained from the analysis in subdomain $D_{2}$. In this case, more than $80 \%$ of the energy is contained in the first mode alone, and the second mode amounts only to $15 \%$. The energy contained in the other modes is comparatively very small. The frequencies associated with the POD coefficients show that modes 1 and 2 are related to $S t=0.054$ (considered above as the sub-harmonic), while modes 3 and 4 peak at St $=0.108$. The interpretation of the apparently contradictory results will be discussed later, in view of the POD modes. 

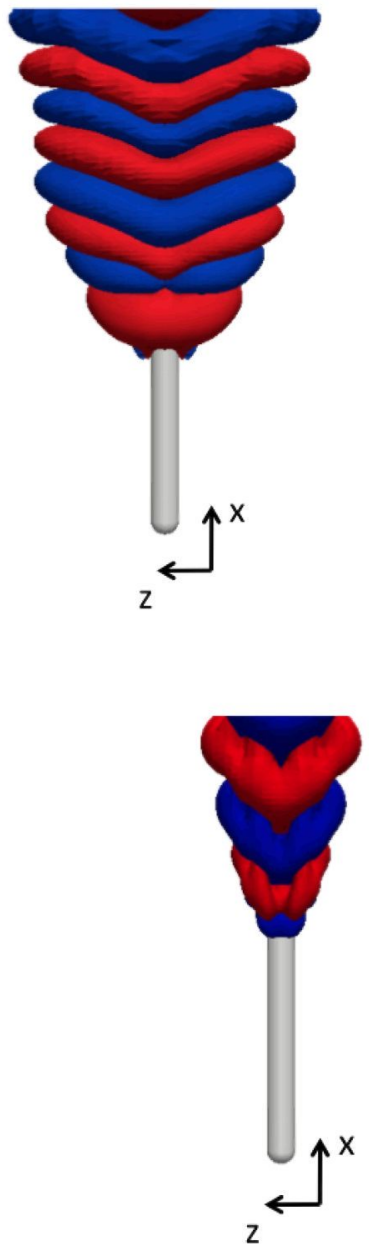

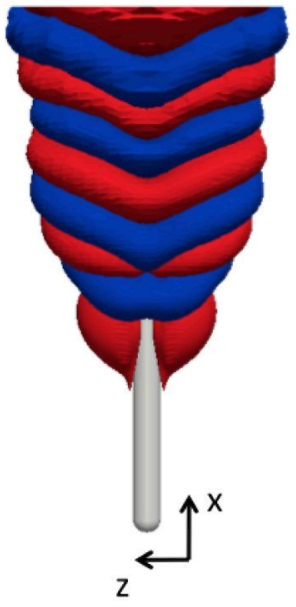

(a) 1 st POD mode $(\mathrm{St}=0.108)$

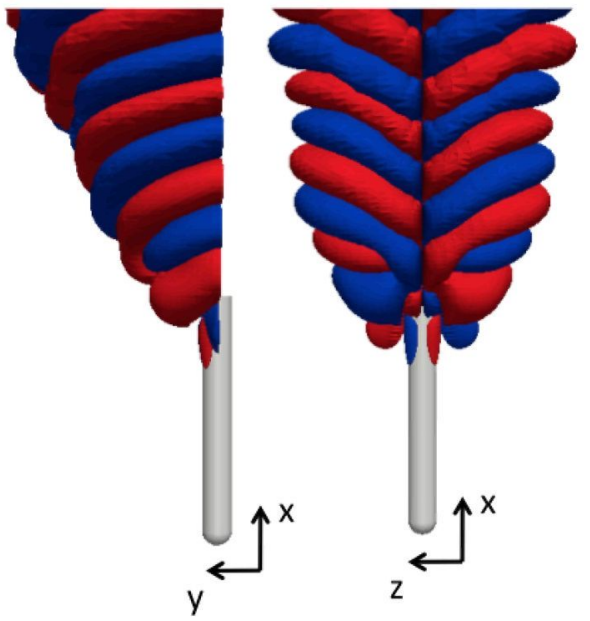

y

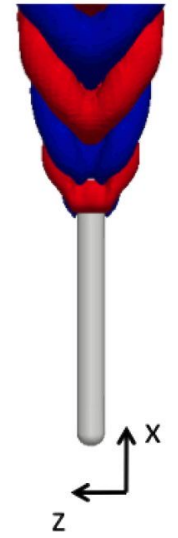

(b) 3rd POD mode $(\mathrm{St}=\mathbf{0 . 1 0 8})$

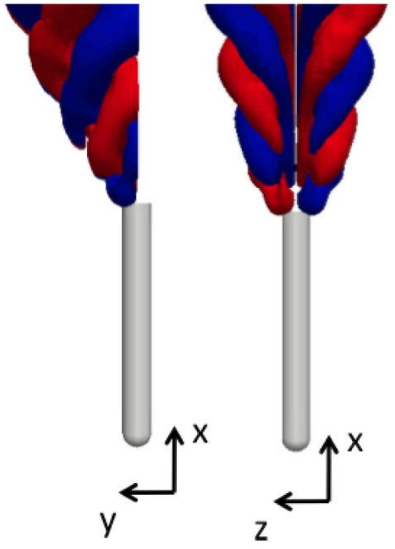

z

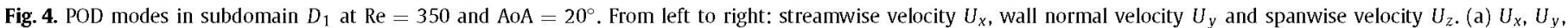

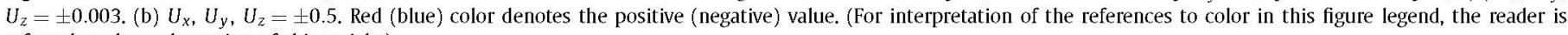
referred to the web version of this article.)

The results of the DMD analysis applied to subdomains $D_{1}$ and $D_{2}$ are shown in Fig. 3(c). As mentioned before, the DMD eigenspectra recovered for all the cases considered in this work comprised only neutral modes with equispaced frequencies. The difference between consecutive frequencies matches exactly the frequency bin dictated by the discrete Fourier transform theory. This serves as additional proof that the DMD results are converged with respect to the number of snapshots. Fig. 3(c, left) presents the results obtained for $D_{1}$. The mean flow is recovered by a steady eigenmode with the largest norm (not shown in the figure). The fundamental frequency $\mathrm{St}=0.108 \simeq 0.11$ has been captured as the dominant DMD mode. Two more frequencies are found also relevant: the approximated sub-harmonic of the fundamental frequency St $=0.649$ and $\mathrm{St}=0.175$. The small mismatches with the frequencies captured in the POD analysis are attributed to the different number of snapshots used in the two cases (see Table 2). When the DMD analysis is applied to the subdomain $D_{2}$ (Fig. 3(c, left)), a higher amplitude is found for the frequency $\mathrm{St}=0.0649$ than for St $=0.108$, consistent with the results of the POD analysis on the same subdomain.

The spatial structure of the first and third POD modes computed in subdomain $D_{1}$ is shown in Fig. 4 . The modes resemble typical wake-instability oscillations, similar to those found in the literature [5,39]. The structure of POD modes 2 and 4 (not shown) is identical to those of modes 1 and 3 , respectively, but with a phase shift. The oscillatory structure in the wake presents a nearly constant wavelength, that can be extracted visually from the figures. This wavelength is approximately the double size in mode 3 (corresponding to $\mathrm{St}=0.057$ ) than in mode 1 (corresponding to $\mathrm{St}=0.108$ ). This finding is reasonable, implying the same convection speed for the two structures. The POD modes also show that the dominant fluctuations existing in the flow, which account for most of the kinetic energy, correspond to oscillations in the afterbody wake and have a limited influence in the upstream region.

Fig. 5 shows the corresponding first and third POD modes computed for subdomain $D_{2}$, that does not include the wake. The spatial structures are concentrated towards the hemisphere-cylinder base, coincident with the end of the subdomain. As was shown in the POD modes for subdomain $D_{1}$, the oscillations upstream of the wake are comparatively very weak, and the structures decay in the upstream direction in a very abrupt manner. Consequently, the portion of the wake fluctuations that is retained in subdomain $D_{2}$ is very small, and the POD and DMD techniques capture the sub-harmonic frequency oscillations as the dominant structures. As illustrated in the figures, the sub-harmonic frequency is associated with a larger wavelength and its upstream influence is more pronounced, leading to a stronger imprint on subdomain $D_{2}$. However, even the structure associated with the sub-harmonic oscillations cannot be recovered in a robust manner if the wake region is eliminated, as the energy-containing fluctuations are also removed. This explains the absence of pairs of POD modes with similar energy content for subdomain $D_{2}$. 

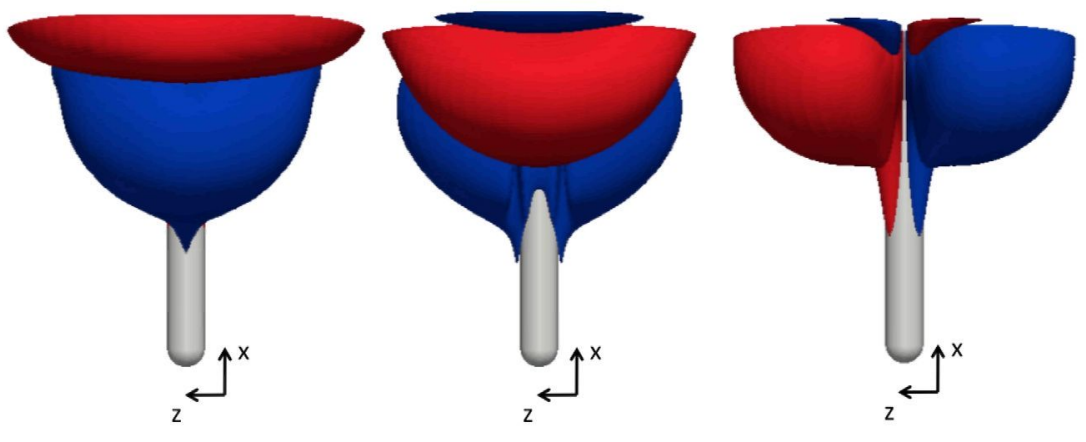

(a) $1 \mathrm{st}$ POD mode $(\mathrm{St}=0.057)$
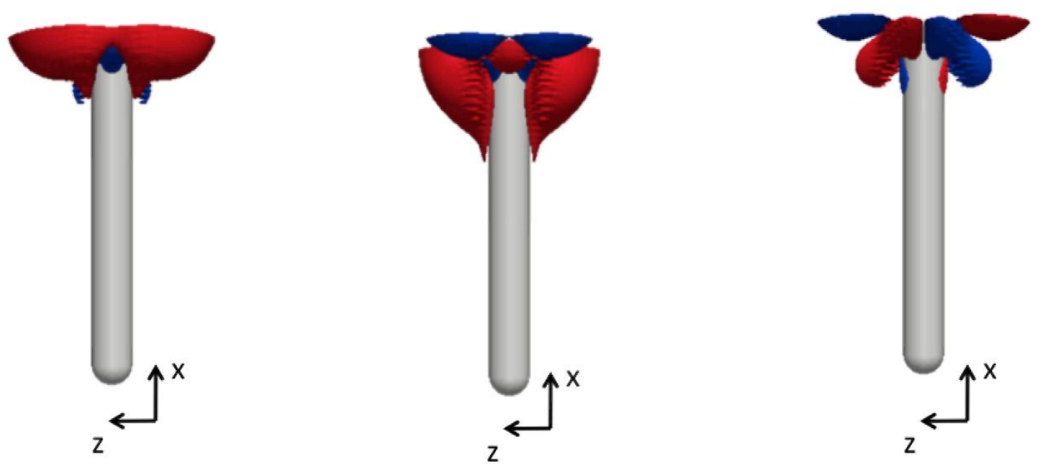

(b) 3rd POD mode $(\mathrm{St}=0.108)$

Fig. 5. POD modes in subdomain $D_{2}$ at $\operatorname{Re}=350$ and AoA $=20^{\circ}$. From left to right: streamwise velocity $U_{x}$, wall normal velocity $U_{y}$ and spanwise velocity $U_{z}$. (a) $U_{x}, U_{y}$, $U_{z}= \pm 0.03$. (b) $U_{x}, U_{y}, U_{z}= \pm 0.5$. Red (blue) color denotes the positive (negative) value. (For interpretation of the references to color in this figure legend, the reader is referred to the web version of this article.)

POD

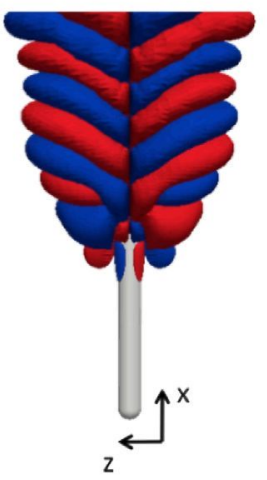

(a) Subdomain $D_{1}$
DMD

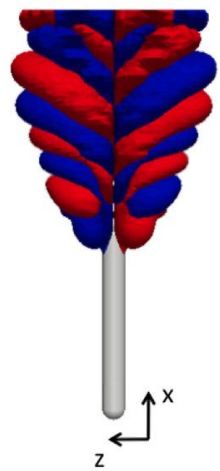

POD
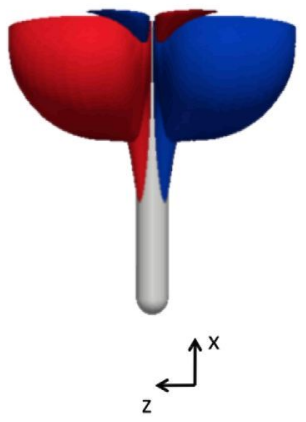

DMD
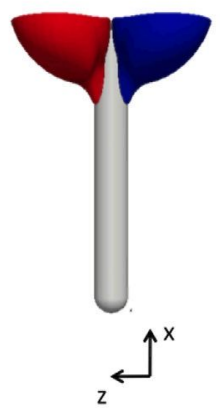

(b) Subdomain $D_{2}$

Fig. 6. Comparison of POD and DMD modes in subdomains $D_{1}$ and $D_{2}$ at $\operatorname{Re}=350$ and $A O A=20^{\circ}$. (a) First POD mode and dominant DMD mode (St $\approx 0.12$ ), POD $U_{z}= \pm 0.003$ and DMD $U_{z}= \pm 0.0003$. (b) First POD mode and dominant DMD mode (St $\approx 0.06$ ), POD $U_{z}= \pm 0.03$ and DMD $U_{z}= \pm 0.0003$. Red (blue) color denotes the positive (negative) value. (For interpretation of the references to color in this figure legend, the reader is referred to the web version of this article.)

Fig. 6 compares POD and DMD modes. As it was expected from this analysis, the modes obtained with these two techniques are very similar.

\subsection{Case 2: $\operatorname{Re}=1000, A \circ A=20^{\circ}$}

Fig. 7 shows instantaneous iso-surfaces of $Q=1000$ for the hemisphere-cylinder flow at $\mathrm{Re}=1000$. Some qualitative differences can be observed with respect to the observations at $\mathrm{Re}=$ 350. The separated shear layer defining the nose separation bubble shows the appearance of a strong spanwise vortex, that can be related to the topological changes in the wall streamtraces that occur as Reynolds number is increased [19]. The lee-side vortices are

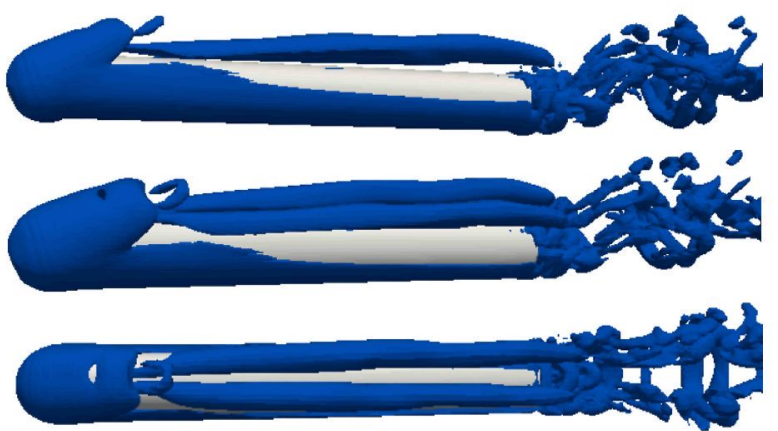

Fig. 7. Iso-surfaces of instantaneous $Q=1000$ at $\operatorname{Re}=1000$ and $\mathrm{AoA}=20^{\circ}$. 

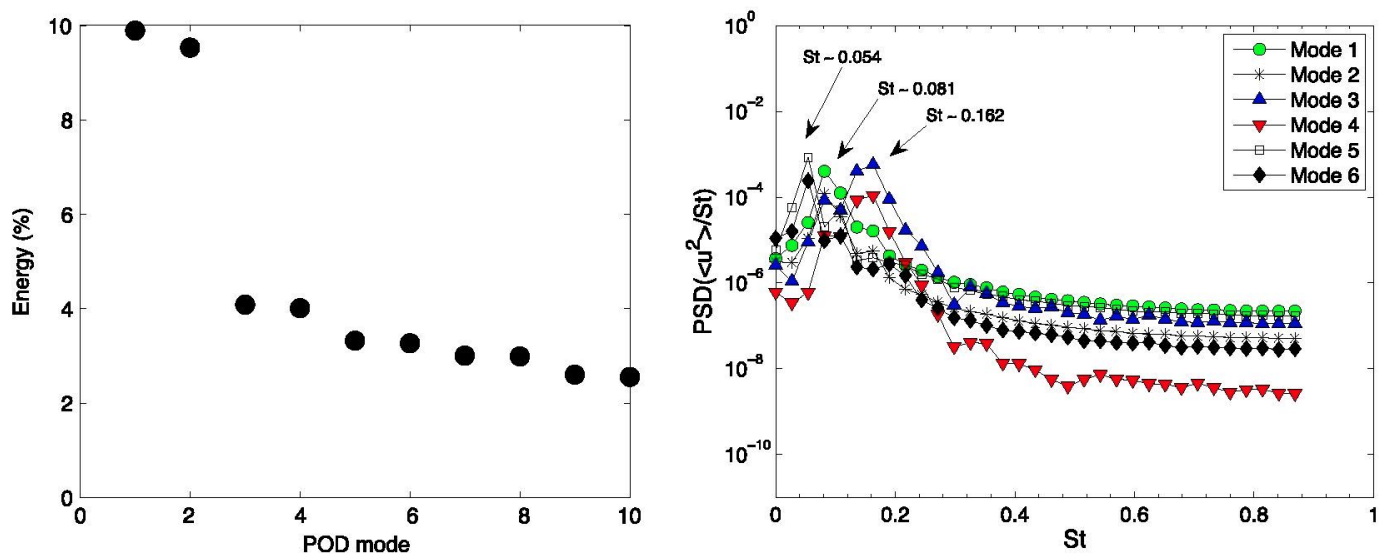

(a) Subdomain $D_{1}$
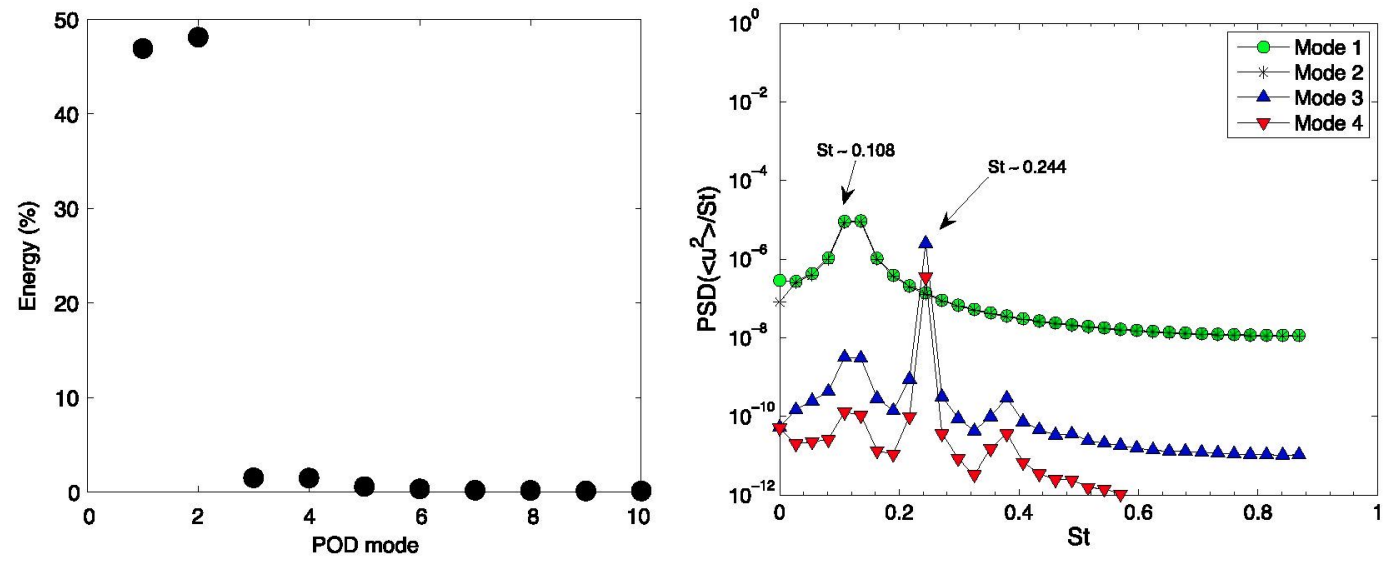

(b) Subdomain $D_{2}$
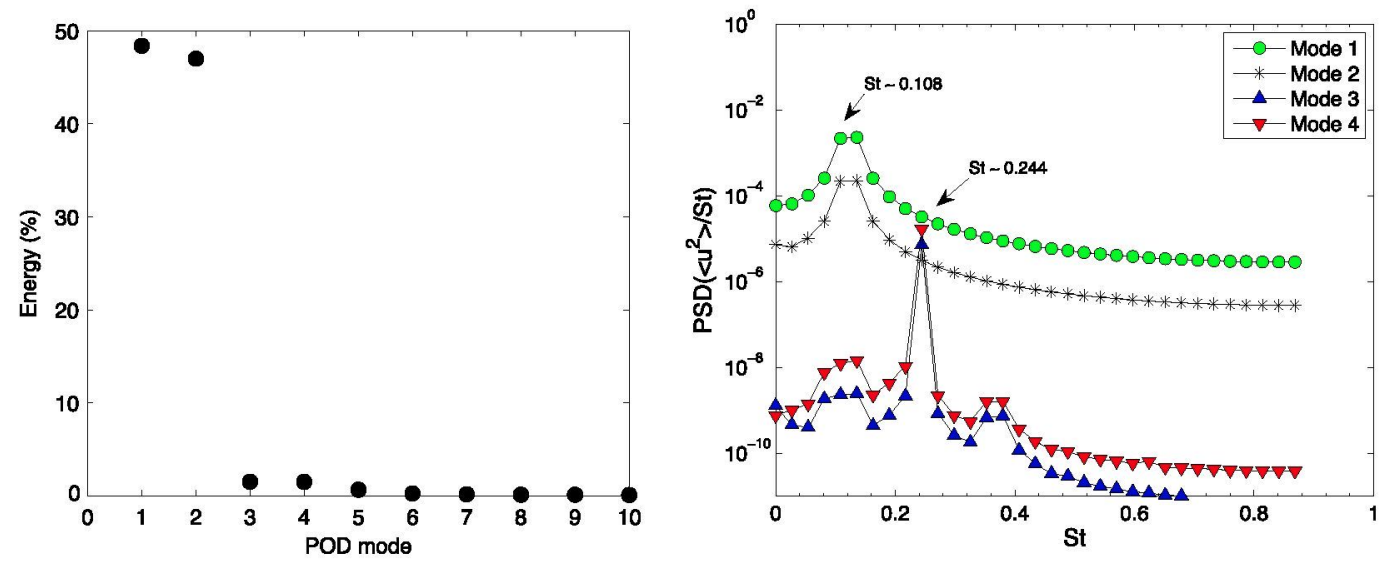

(c) Subdomain $D_{3}$

Fig. 8. POD analysis in domains $D_{1}, D_{2}$ and $D_{3}$ at $\mathrm{Re}=1000$ and $\mathrm{AoA}=20^{\circ}$. Kinetic energy distribution (left) and PSD of POD coefficients (right).

defined more clearly at this higher Reynolds number, and exhibit a peristaltic oscillation along the streamwise direction that can be identified in the figure. This peristaltic motions have been related to the primary instability of the counter-rotating vortex pair conforming the wake of a three-dimensional bluff-body [4,11]. Finally, a complex flow pattern is observed in the wake region.

POD and DMD analyses have been performed in this case in subdomains $D_{1}, D_{2}$ and $D_{3}$. A preliminary analysis of this case employing subdomain $D_{3}$ was presented in Le Clainche et al. [20]. Fig. 8 compares the relative kinetic energy content for the 10 leading POD modes (left) and the power spectra for the leading six POD modes (right), corresponding to the analyses done in subdo- mains $D_{1}(\mathrm{a}), D_{2}$ (b) and $D_{3}$ (c). The energy contained in the two leading POD modes for $D_{1}$ amounts to nearly $20 \%$ of the total. However, the accumulated energy content of the following 8 POD modes is approximately the same as for the first two modes. The frequency spectrum for this case shows no clear prevalence of any frequency, but rather a broadband oscillation centered on $\mathrm{St}=0.1$. On the other hand, when the afterbody wake region is removed (subdomain $D_{2}$ ), the first couple of POD modes account for more than $90 \%$ of the energy, and correspond to a clearly defined frequency St $\approx 0.108$. Modes 3 and 4 correspond to the harmonic frequency St $=0.244$, but their energy content is much smaller to be considered relevant here. It should be remarked that, with 

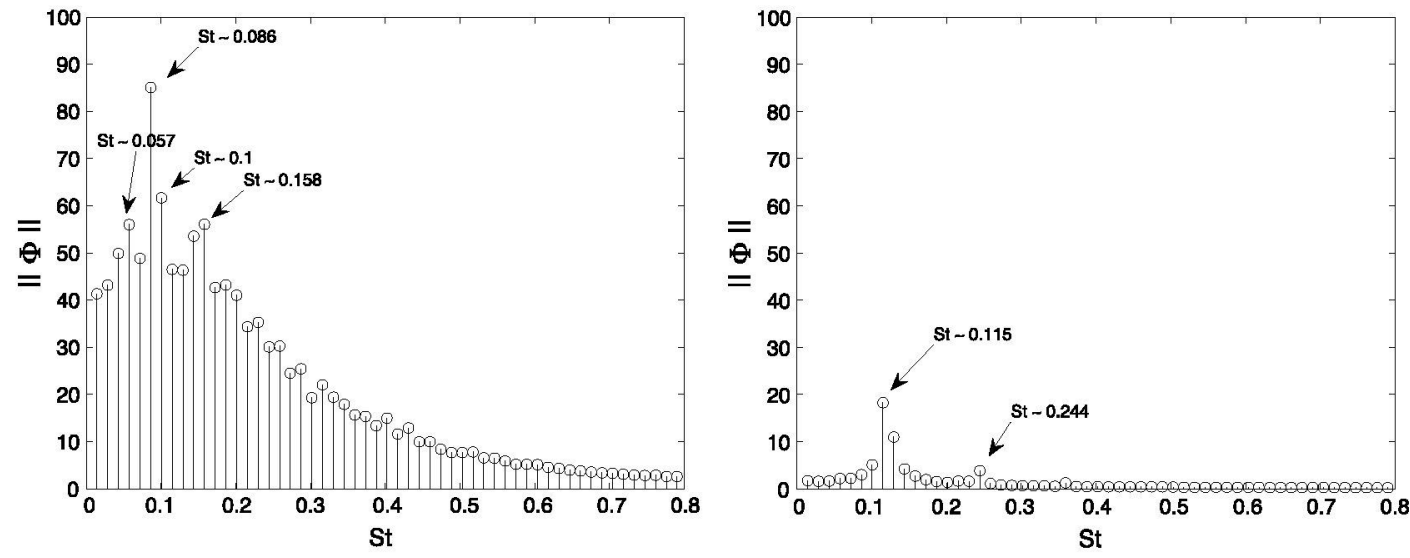

(a) Subdomains $D_{1}$ (left) and $D_{2}$ (right).
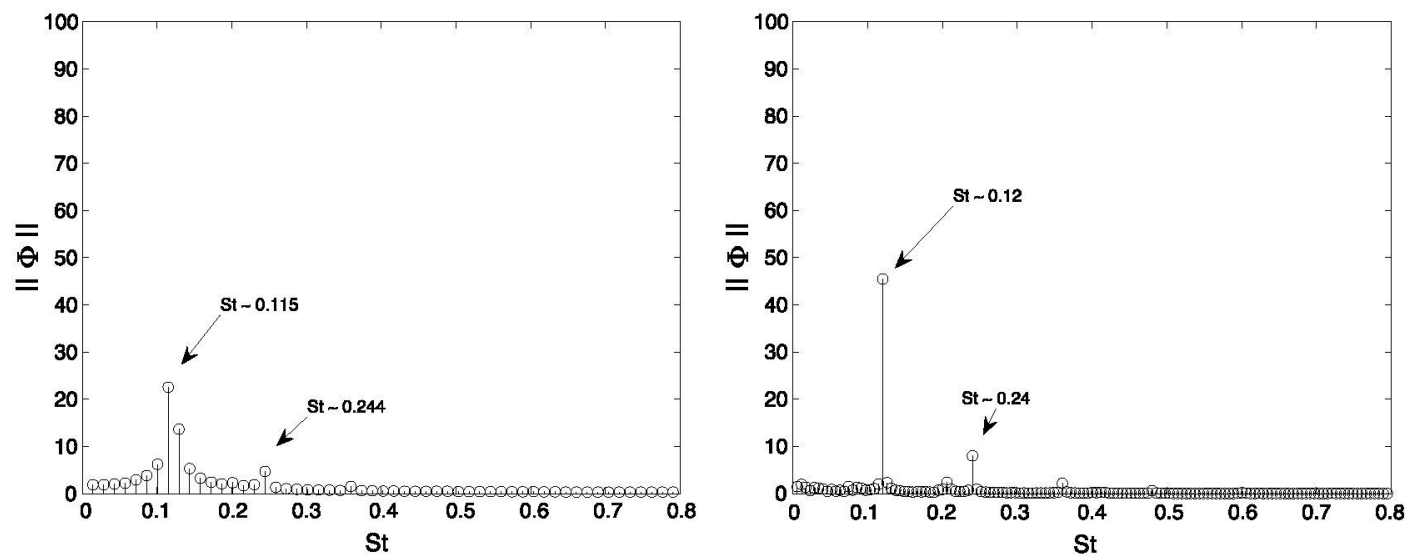

(b) Subdomain $D_{3}$, with $N=120$ (left) and $N=300$ (right) snapshots.

Fig. 9. DMD frequency diagram in subdomains $D_{1}, D_{2}$ and $D_{3}$ at $\operatorname{Re}=350$ and $A \circ A=20^{\circ}$. Frequency spectrum in $D_{1}$ (a, left); Frequency spectrum in $D_{2}$ (a, right); Frequency spectrum in $D_{3}: 120$ snapshots (b, left) and 300 snapshots (b, right).

removing the spatial region containing the wake, the broadband behavior of the spectra also disappears. This shows that the fluctuations in the wake at this Reynolds number are already in a transitional state, but the oscillations associated with the separated flow on the lee-side of the cylinder are mostly laminar, and characterized by a single frequency. When reducing the POD domain further to subdomain $D_{3}$, the POD spectrum is nearly identical than the one corresponding to $D_{2}$.

Fig. 9 compares the dominant frequencies obtained with DMD applied to domains $D_{1}, D_{2}$ and $D_{3}$. The results follow the same interpretation as the POD analyses. A broad spectrum of frequencies is recovered for subdomain $D_{1}$, which is centered on St $=$ 0.1 and decays slowly towards higher frequencies. This broadband spectrum is again attributed to the transitional wake oscillations, as it disappears when the computational domain is truncated to subdomain $D_{2}$. For $D_{2}$, a well-defined peak at St $=0.115$ and a first harmonic of small energy at St $=0.244$ are recovered. It is also worth noting that the energy content on the DMD modes, calculated following (11), is much smaller than that for $D_{1}$. Fig. $9(\mathrm{~b}$, left) shows the result of performing DMD analysis on domain $D_{3}$ employing the same number of snapshots $(N=120)$ and $\Delta t$ as for the $D_{1}$ and $D_{2}$ cases; the frequency spectrum is nearly identical to that for $D_{2}$.

The use of the reduced domain $D_{3}$ permits increasing the number of snapshots employed in the DMD analysis, thus reducing the width of the frequency bins in which the DMD modes appear, in the same manner as predicted for a discrete Fourier transform. After increasing the number of snapshots from $N=120$ to $N=300$, the frequency spacing is reduced from $\Delta S t=0.013$ to 0.0057 ; the resulting DMD spectrum is shown in Fig. 9(b, right). The refined frequency bin enables the dominant frequencies to be recovered as well-definite tonal peaks with higher energy levels. The fundamental frequency is accurately determine now as $\mathrm{St}=0.12$, a small number of harmonics ( $S t=0.24,0.36,0.48$ ) can also be identified.

Fig. 10 shows the POD modes corresponding to the fundamental frequency $\mathrm{St} \simeq 0.11$ calculated in the subdomains $D_{1}, D_{2}$ and $D_{3}$. The mode corresponding to subdomain $D_{1}$ is again dominated by fluctuations in the wake, as happened for $\operatorname{Re}=350$, but this structure is now less clearly defined. However, a new coherent structure is recovered for the same POD modes on the lee-side of the cylinder, extending from the cylinder base up to the nose separation region, in contrast with the results at the lower Reynolds number. As was discussed in Le Clainche et al. [20], the flow topology at $\operatorname{Re}=1000$ is characterized by horn-vortices separation at the nose. Horn vortices leave the body surface perpendicular to it, and then bend towards the downstream direction to merge with the lee-ward vortices. The structure of the POD modes illustrates that, under these conditions, flow oscillations are also observable in the lee-ward vortices upstream of the cylinder base. These structures are recovered in a robust manner also when POD is applied on the reduced domains $D_{2}$ and $D_{3}$, where the more energetic wake fluctuations are removed.

Fig. 11 compares POD and DMD modes in the subdomain $D_{1}$. As it was expected from this analysis, the modes obtained with these two techniques are very similar. POD and DMD modes calculated in subdomains $D_{2}$ and $D_{3}$ are also identical. 

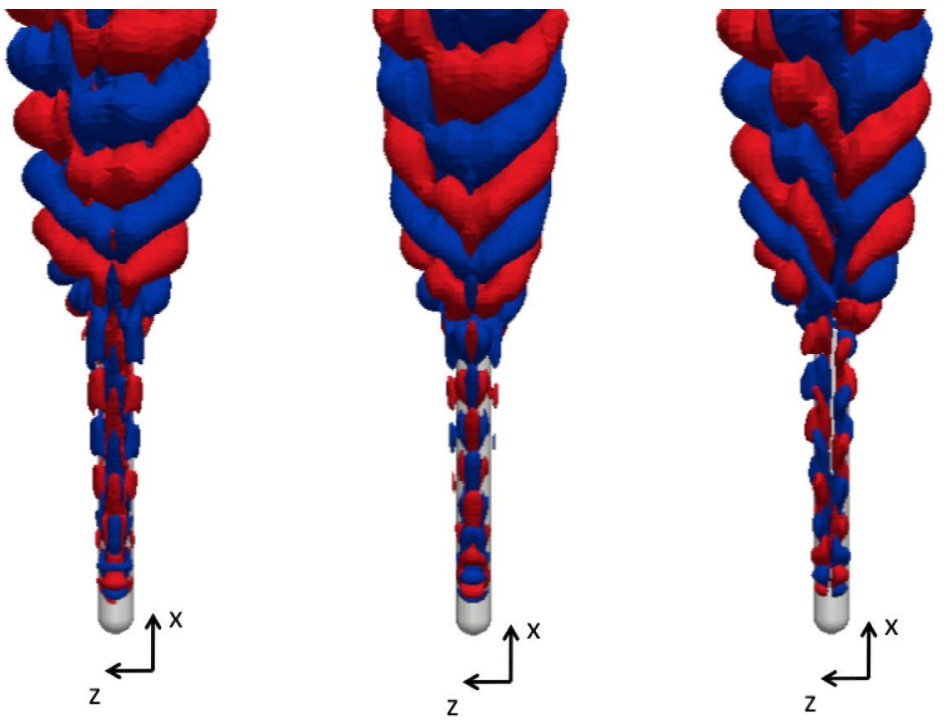

(a) Subdomain $D_{1}$
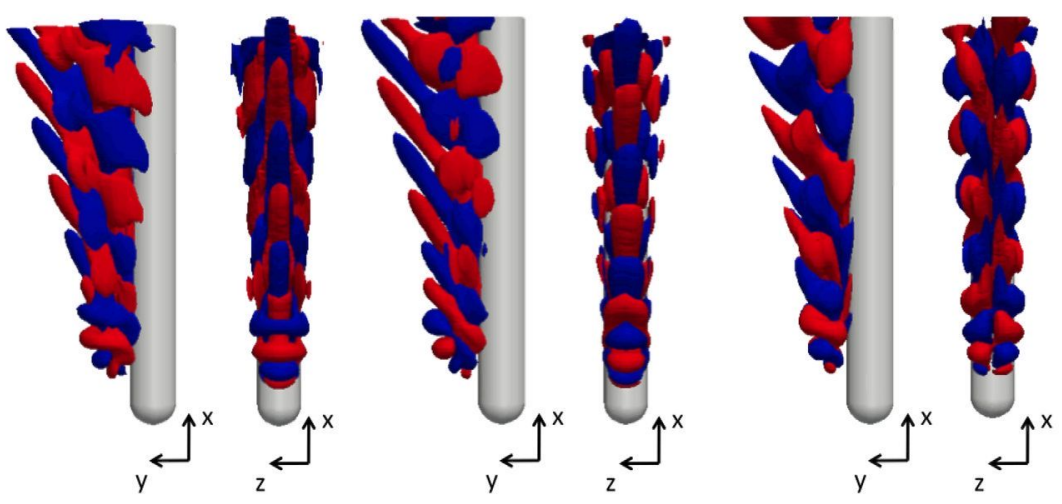

(b) Subdomain $\mathrm{D}_{2}$
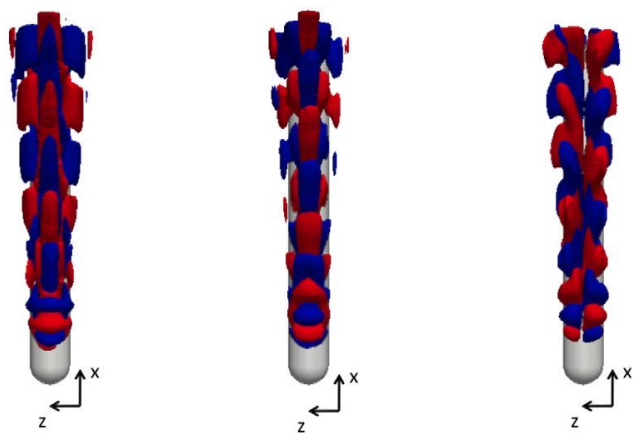

(c) Subdomain $D_{3}$

Fig. 10. POD modes in subdomains $D_{1}, D_{2}$ and $D_{3}$ at $\mathrm{Re}=1000$ and $A \circ A=20^{\circ}$, corresponding to the first POD mode on each case. From left to right: streamwise velocity $U_{x}$, wall normal velocity $U_{y}$ and spanwise velocity $U_{z}$. (a) $U_{x}, U_{y}, U_{z}= \pm 0.5$. (b) $U_{x}, U_{y}, U_{z}= \pm 0.5$. (c) $U_{x}, U_{y}, U_{z}= \pm 0.03$. Red (blue) color denotes the positive (negative) value. (For interpretation of the references to color in this figure legend, the reader is referred to the web version of this article.)

\section{Conclusions}

A data set obtained from three-dimensional direct numerical simulations of the flow around a hemisphere-cylinder at $\mathrm{AoA}=$ $20^{\circ}$ and low Reynolds number has been employed in the extraction and analysis of the dominant coherent structures via proper orthogonal decomposition and dynamic mode decomposition. For this geometry, the flow first becomes unstable between $\mathrm{Re}=300$ and $\operatorname{Re}=350$ [19], giving rise to unsteadiness. Two Reynolds num- bers, namely $\operatorname{Re}=350$ and 1000 , are considered in this analysis. The present work addressed two main questions. The first one is concerned with the ability of both POD and DMD to extract the dominant flow structures and frequencies if small portions or subdomains are used instead of the complete DNS data set. The second question is the determination of the physical mechanism responsible for the unsteadiness in this particular flow, with the aim of shedding light on the primary instability take occurs slightly below $\operatorname{Re}=350$ and, if possible, relating it to the dominant frequencies at $\operatorname{Re}=1000$. 
POD

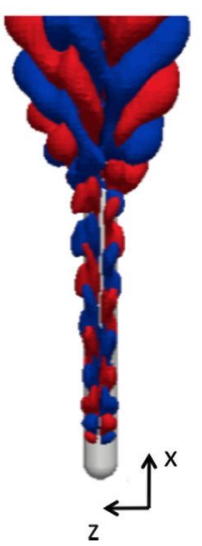

DMD

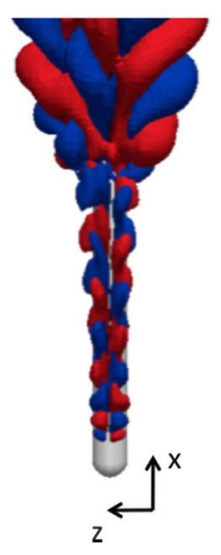

Fig. 11. Comparison of POD and DMD modes in subdomain $D_{1}$ at $\operatorname{Re}=1000$ and AoA $=20^{\circ}$. First POD mode and dominant DMD mode (St $\approx 0.1$ ), POD $U_{z}= \pm 0.003$ and DMD $U_{z}= \pm 0.0003$. Red (blue) color denotes the positive (negative) value. (For interpretation of the references to color in this figure legend, the reader is referred to the web version of this article.)

Most often, the memory requirements associated with these methodologies are prohibitive if time-resolved three-dimensional simulation data is to be used. In these cases, it is necessary to downsample the data to lower spatial resolutions and/or to extract subdomains, in order to make the problem solvable. It can also be thought of extracting and analyzing a particularly interesting region, isolating it from the fluctuations in the rest of the domain. This work considered three spatial subdomains of the complete domain in which the DNS computations were performed. The first one was chosen in order to contain all the flow fluctuations, while reducing the DMD size problem to something tractable in the machine available. This subdomain included all the afterbody wake computed. The second subdomain was the result of truncating the first subdomain to exclude the wake oscillations. Only fluctuations existing on the lee-side of the cylinder are then maintained. The third subdomain is similar to the second one, but its extent is decreased to enable use of a higher number of snapshots, refining the temporal resolution of DMD.

The present results show that the onset of unsteadiness is due to an instability of the three-dimensional wake, in a manner analogous to related results in the literature $[5,14,39]$. The oscillations are initiated as a peristaltic instability $[4,11]$ of the pair of counterrotating vortices conforming the wake, with a dominant frequency St $\approx 0.11$. The fluctuations commence close to the cylinder base and are convected downstream, and consequently their influence upstream of the base are very small. The POD and DMD analyses at $\operatorname{Re}=350$ capture both the spatial structure of the fluctuations and the fundamental frequency and its sub-harmonic, as far as the wake region is included in the analysis. The leading couple of POD modes, related to the fundamental frequency, account for more than $50 \%$ of the fluctuating kinetic energy. The second couple, related to the sub-harmonic, accounts for another $24 \%$. If the wake is removed from the analysis, the imprint of the fluctuations on the lee-side of the cylinder is so weak that the POD and DMD fail to deliver robust and consistent results.

At $\operatorname{Re}=1000$, the oscillations related to the wake are of much higher amplitude, and three-dimensional vortex shedding occurs already at the cylinder base (see Fig. 1 in Le Clainche et al. [20]). The POD and DMD analyses applied to the subdomain including the wake show a broadband spectrum with maximum around $\mathrm{St}=$ 0.11 , that decays slowly for higher frequencies. A large number of POD modes have comparable energy and their respective PSD do not show a distinct frequency. However, when the wake region is removed from the analysis, the resulting POD analysis show the existence of a pair of POD modes accounting for nearly $90 \%$ of the energy, and with a clearly defined frequency at St $=0.11$. DMD results accurately reproduce this observation. Visual inspection of the POD modes serves to explain this apparent controversy. The first POD mode computed for the subdomain including the wake has the strongest fluctuations in the wake, as was the case for $\operatorname{Re}=350$; but as opposed to it, also contains non-negligible structure on the lee-side of the cylinder, in the region where the horn/leeward vortices lie. The observable wavelengths in the cylinder region and in the wake region are different, denoting different convection speeds of the structures on the two regions. When the POD/DMD analyses are performed in a reduced subdomain that does not contains the wake, the large amplitude oscillations related to the vortex shedding are removed from the analysis, and only the low-amplitude oscillations of the laminar leeward vortices are retained. It is worth of mention that the spatial structure of the oscillations at the fundamental frequency over the cylinder is accurately recovered even if the wake region is not removed.

Finally, the experience gained in the use of POD and DMD techniques illustrates some aspects that should be borne in mind for their application. Some prior knowledge or understanding of the spatial locations where strong oscillations are present need to be attained before analyzing subdomains. POD and DMD can deliver fundamentally different results when applied to different subdomains within the same DNS data set. The POD and DMD formulations employed here rank the respective modes according to the integrated kinetic energy in the domain considered. If the regions containing most of the fluctuating kinetic energy is removed, as was done for $\operatorname{Re}=350$, the analysis done in a reduced domain would lead to inconsistent and misleading results. On the other hand, truncation and extraction of subdomains can be used to focus the analysis on existing coherent structures, filtering out fluctuations that are more energetic but are separated in space. This approach was used here for $\operatorname{Re}=1000$, to separate the tonal-peak laminar oscillations in the horn/leeward vortices over the cylinder from the more energetic broadband vortex shedding at the wake. However, we have to keep in mind that, besides we can separate these two kinds of fluctuations in the analysis, the physical origin of them might be the same: it cannot be ascertained that the horn/leeward vortices oscillations and the wake vortex shedding are independent phenomena or they are generated by the same peristaltic instability found for $\mathrm{Re}=350$. In any case, both the afterbody wake and the leeward vortices over the cylinder present a counter-rotating vortex pair instability, which is dominated by the same St $\approx 0.11$ frequency.

\section{Conflict of interest statement}

None declared.

\section{Acknowledgements}

Support of the Marie Curie Grant PIRSES-GA-2009-247651 "FP7-PEOPLE-IRSES: ICOMASEF - Instability and Control of Massively Separated Flows" is gratefully acknowledged. Support of the Spanish Ministry of Science and Innovation through Grant TRA2012-34148 "Mejoras del Rendimiento aerodinámico de alas mediante control de mecanismos de inestabilidad global" is gratefully acknowledged. The work of D.R. was funded by the Marie Curie - COFUND programme. Support of the Defense Science and Technology Organization of Australia is gratefully acknowledged. This work was supported by the Multi-modal Australian ScienceS Imaging and Visualization Environment (MASSIVE) (www.massive.org.au). 


\section{References}

[1] S. Bagheri, Koopman-mode decomposition of the cylinder wake, J. Fluid Mech. 726 (2013) 596-623.

[2] D. Barkley, R.D. Henderson, Three-dimensional Floquet stability analysis of the wake of a circular cylinder, I. Fluid Mech. 322 (1996) 215-241.

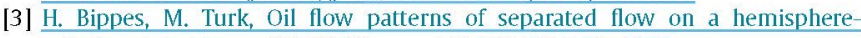
cylinder at incidence, DFVLR-FB report IB 222-83 A 07, 1983.

[4] P. Bohorquez, L. Parras, Three-dimensional numerical simulation of the wake flow of an afterbody at subsonic speeds, Theor. Comput. Fluid Dyn. 27 (1-2) (2013) 201-218.

[5] P. Bohorquez, E. Sanmiguel-Rojas, A. Sevilla, J. Jimenez-Gonzalez, C. MartínezBazán, Stability and dynamics of the laminar wake past a slender blunt-based axisymmetric body. J. Fluid Mech. 676 (2011) 110-144.

[6] K.K. Chen, J.H. Tu, C. Rowley, Variants of dynamic mode decomposition: boundary condition, Koopman, and Fourier analyses, I. Nonlinear Sci. 22 (6) (2012) $887-915$.

[7] C.E. Costis, D.P. Telionis, Unsteady vortical wakes over a prolate spheroid, AIAA J. $20(1983)$ 1189-1193.

[8] S.C. Crow, F.H. Champagne, Orderly structure in jet turbulence, J. Fluid Mech. 48 (3) (1971) 547-591.

[9] B. Fairlie, Flow separation on bodies of revolution at incidence, in: 7th Australian Hydraulics and Fluid Mechanics Conference, Brisbane, 18-22 August, 1980.

[10] M. Gaster, E. Kit, I. Wygnansky, Large-scale structures in a forced turbulent mixing layer, J. Fluid Mech. 150 (1985) 23-39.

[11] B. Ghidersa, I. Dusek, Breaking of axisymmetry and onset of unsteadiness in the wake of a sphere, I. Fluid Mech. 423 (2000) 33-69.

[12] F. Gómez, S. Le Clainche, P. Paredes, M. Hermanns, V. Theofilis, Global linear instability at the dawn of its 4 th decade: recent progress and remaining challenges, AIAA J. 50 (12) (2012) 2731.

[13] A. Gross, C. Jagadeesh, H. Fasel, Numerical investigation of three-dimensional separation on axisymmetric bodies at angle of attacke, AIAA paper 2012-0098, 2012.

[14] K. Gumowski, J. Miedzik, S. Goujon-Durand, P. Jenffer, J. Wesfreid, Transition to a time-dependent state of fluid flow in the wake of a sphere, Phys. Rev. E 77 (5) (2008) 055308.

[15] N. Hoang, O. Rediniotis, D. Telionis, Symmetric and asymmetric separation patterns over a hemisphere cylinder at low Reynolds number and high incidences, J. Fluids Struct. 11 (1997) 793-817.

[16] P. Holmes, I. Lumley, G. Berkooz, Turbulence Coherent Structures, Dynamical Systems and Symmetry, Cambridge University Press, 1996.

[17] T. Hsieh, An investigation of separated flow about a hemisphere-cylinder at 0 to $19 \mathrm{deg}$ incidence in the Mach number range from 0.6 to $1.5,1974$, AEDCTR-76-112.

[18] J. Hunt, A. Wray, P. Moin, Eddies, stream, and convergence zones in turbulent flows, Center for turbulence research report, 1988, p. 193.

[19] S. Le Clainche Martínez, Instability and topology bifurcations on a hemispherecylinder at high angle of attack, PhD thesis, Universidad Politécnica de Madrid, School of Aeronautics, 2013.
[20] S. Le Clainche, J.I. Li, V. Theofilis, I. Soria, Flow around a hemisphere-cylinder at high angle of attack and low Reynolds number. Part I: Experimental and numerical investigation, Aerosp. Sci. Technol. 44 (2015) 77-87, in this issue.

[21] E. Merzari, H. Ninokata, A.M.R. Mahmood, Proper orthogonal decomposition of the flow in geometries containing a narrow gap, Theor, Comput. Fluid Dyn, 23 (2009) 333-351.

[22] I. Mezic, Spectral properties of dynamical systems, model reduction and decompositions, Nonlinear Dyn. 41 (2013) 309-325.

[23] I. Mezic, Analysis of fluid flows via spectral properties of the Koopman operator, Annu. Rev. Fluid Mech. 45 (2013) 357-378.

[24] B. Noack, K. Afanasiev, M. Morzynski, G. Tadmor, F. Thiele, A hierarchy of low dimensional models for the transient and post-transient cylinder wake, J. Fluid Mech. 497 (2003) 335-363.

[25] K. Oberleithner, M. Sieber, C. Nayeri, C. Paschereit, C. Petz, H. Hege, B. Noack, L. Wygnanski, Three-dimensional coherent structures in a swirling jet undergoing vortex breakdown: stability analysis and empirical mode construction, J. Fluid Mech. 679 (2011) 383-414.

[26] M. Rancic, J. Purser, F. Mesinger, A global-shallow water model using an expanded spherical cube, Q. I. R. Meteorol. Soc, 122 (1996) 959-982.

[27] D. Rempfer, H.F. Fasel, Evolution of three-dimensional coherent structures in a flat-plate boundary laver, J. Fluid Mech. 260 (1994) 351-357.

[28] D. Rempfer, H.F. Fasel, Dynamics of three-dimensional coherent structures in a flat-plate boundary layer, J. Fluid Mech. 275 (1994) 257-283.

[29] C.W. Rowley, T. Colonius, R.M. Murray, Pod based models of self-sustained os cillations in the flow past an open cavity, AIAA Paper 2000-1969, 2000.

[30] C. Rowley, T. Colonius, R. Murray, Model reduction for compressible flows using pod and Galerkin projection, Physica D 189 (2004) 115-129.

[31] C.W. Rowley, I. Mezic, S. Bagheri, P. Schlatter, D. Henningson, Spectral analysis of nonlinear flows, I. Fluid Mech. 641 (2009) 115-127.

[32] E. Sanmiguel-Rojas, J.I. Jimênez-Gonzâlez, P. Bohorquez, G. Pawlak, C. MartînezBazân, Effect of base cavities on the stability of the wake behind slender bluntbased axisymmetric bodies, Phys. Fluids 23 (2011) 114103.

[33] E. Sanmiguel-Rojas, C. Martínez-Bazán, J.M. Chomaz, Global mode analysis of axisymmetric bluff-body wakes: stabilization by base bleed, Phys. Fluids 21 (2009) 114102.

[34] P.J. Schmid, Dynamic mode decomposition of numerical and experimental data, I. Fluid Mech. 656 (2010) 5-28.

[35] L. Sirovich, Turbulence and the dynamics of coherent structures, Parts I-III, Q. Appl. Math. 45 (1987) 561.

[36] O. Tammisola, F. Lundell, P. Schlatter, A. Wehrfritz, L.D. Soderberg, Global linear and nonlinear stability of viscous confined plane wakes with co-flow, J. Fluid Mech. 675 (2011) 397-434.

[37] Tapan K. Sengupta, N. Singh, V. Suman, Dynamical system approach to instability of flow past a circular cylinder, J. Fluid Mech. 656 (2010) 82-115.

[38] M. Tobak, D. Peake, Topology of two-dimensional separated flows, Annu. Rev. Fluid Mech. 14 (1992) 61-85.

[39] A.G. Tomboulides, S.A. Orszag, Numerical investigation of transitional and weak turbulent flow past a sphere, J. Fluid Mech, 416 (2000) 45-73. 